\title{
Diagnostic Imaging in Patients with Spinal Injury: an Optimized Procedure Based on Novel Technologies
}

\author{
DOI: 10.17691/stm2018.10.2.14
}

Received October 16, 2017

A.V. Bazhin, MD, PhD, Radiologist, Department of Radiology';

E.A. Egorova, MD, DSc, Professor, Department of Diagnostic Radiology ${ }^{2}$

${ }^{1}$ City Clinical Hospital named after S.S. Yudin, Moscow Healthcare Department, 4 Kolomensky Proezd, Moscow, 115446, Russia;

${ }^{2}$ Moscow State University of Medicine and Dentistry named after A.I. Evdokimov, 20/1 Delegatskaya St., Moscow, 127473, Russia

The aim of the study was to optimize the procedure of diagnostic imaging in patients suffering from spinal and spinal cord injuries by using novel technologies and modalities of diagnostic radiology.

Materials and Methods. Total of 105 patients with spinal cord injuries were included in the study. Of them, $32.4 \%$ of cases resulted from traffic accidents and $24.3 \%$ - from falls from heights $>1 \mathrm{~m}$ with significant axial loading. The predominant injury components were compression $(n=51,48.5 \%)$ and flexure $(n=25,23.8 \%)$ lesions, and the rarest were extensor lesions $(n=6)$, extensor lesions with discoligament tears caused by dislocations $(n=5)$ or excessive stretching $(n=3)$. Clinical and anamnestic data, neurological status (International Standards for Neurological Classification of Spinal Cord Injury, ASIA), and laboratory results were obtained. The imaging tests included spine X-ray and the novel radiology techniques - multislice computed tomography and magnetic resonance imaging.

Results. According to our findings, the examination protocol in patients with spine and spinal cord injuries should be based on the qualitative and quantitative characteristics of the lesions, the presence of neurological symptoms and signs of instability. In the cases of multiple multilevel and combined injuries of the spine and spinal cord and in the case of a severe patient's condition, the immediate imaging approach should be limited to a spine X-ray test; upon patient's stabilization, multislice computed tomography according to the "Polytrauma" (Whole body) protocol should be performed, including intravenous contrast enhancement. Magnetic resonance imaging should be performed in the early and late periods according to clinical indications.

Key words: spine injuries; spinal trauma; polytrauma; spine X-ray; multislice computed tomography; magnetic resonance imaging.

\section{Introduction}

Spine injuries account for $5-20 \%$ of all lesions of the skeleton $[1,2]$.

There are three types of damage:

1) uncomplicated trauma of the vertebral column without damage to the neurovascular elements of the spinal canal;

2) spinal cord injury - without spinal injury;

3) spinal cord and vertebrae injury (SCVI) - a combination of damage to the structures of the spine and the neurovascular formations inside the spinal canal.

In $20-50 \%$ of patients, multilevel spine injuries are found, and in $13-63 \%$ of cases - SCVI [3-5].

Spinal cord and vertebrae injury involves mechanical damage to the spine and the contents of the spinal canal (spinal cord, its membranes and vessels, spinal nerves). In more than $50 \%$ of cases, SCVI is observed in patients younger than 40 years old, of whom $75 \%$ are males (National Spinal Cord Injury Statistical Center, The University of Alabama, USA, 2015). Such injuries are associated with complications, disability, and a high mortality rate. In our country, each year up to $37 \%$ of people with SCVI die at the prehospital stage [6]; the mortality rate in hospitalized SCVI patients reaches $58.3 \%$ [7].

In the first $72 \mathrm{~h}$, patients with severe combined trauma need surgical correction and spine stabilization [1]; timely neurosurgical interventions improve the prognosis in SCVI patients in $22-87 \%$ of cases. Death of patients in a later period is caused mostly by secondary complications, largely by an ascending urinary infection, pressure ulcers, and hypostatic pneumonia [6].

The localization, mechanisms, and nature of SCVI are essential for radiologists and clinicians. These factors determine the diagnostic procedure (study area, projections, and protocols) and underlie the trauma classification into uncomplicated spine trauma, cerebrospinal trauma or SCVI.

Correct and well-based classification plays an important role in the further treatment strategy. There are different principles of classifications of spine and spinal cord injuries that serve different purposes:

ICD-10 (1990) - developed for medical and

Corresponding author: Elena A. Egorova, e-mail: tylsit@mail.ru 
epidemiological purposes, and for assessment of the quality of medical care; it mainly serves to compile medical statistics. The SCVI is listed in the "Injuries, poisonings and some other consequences of external factors (S00-T98)" section;

a classification based on the concept of three mechanical columns [8] and a modified, expanded and in-depth classification by Saldun [9], which have been developed on the basis of localization, nature, and mechanism of the spine lesions, the degree of vertebrae deformation and instability;

the universal classification of fractures (the scale of severity of spinal cord injury) of the Association for Osteosynthesis/Association for the Study of Internal Fixation (AO/ASIF) [10], according to which vertebral lesions are viewed at the level of two support structures (anterior and posterior), taking into account the anatomical and biomechanical characteristics.

According to the AO/ASIF classification, three types of fractures are defined:

Type $A-$ compression of the vertebral body (simple compression, split and explosion fractures due to flexion);

Type $B$ - damage to the front and back support structures due to stretching $\left(B_{\mid}-\right.$damage to the posterior muscle-ligament complex, $B_{\|}$- damage to the bone structures of the posterior complex, $\mathrm{B}_{\mathrm{II}}$ - damage to the anterior complex involving the intervertebral disc);

Type $\mathrm{C}$ - damage to the anterior and posterior support structures because of rotational and translational motion $\left(C_{1}\right.$ - rotation with compression of the vertebral bodies, $\mathrm{C}_{\|}$- stretching of the supporting vertebral columns, $\mathrm{C}_{\mathrm{III}}$ - rotational displacement in combination with a horizontal shift of the fragments).

This classification looks quite complete and consistent. However, in practical use, specialists conducting physical examination, and X-ray, multislice computed tomography (MSCT), or magnetic resonance imaging (MRI) tests, encounter difficulties in making an unequivocal diagnosis. This is due to the fact that vertebral lesions not related to fractures of their bodies are considered; also missed are injuries of other anatomical structures (spinal cord, capsular ligamentous and paravertebral tissues) as well as various complications.

The classification of spine and spinal cord injuries based on the concept of three mechanical columns considers the following factors:

localization;

character and degree of damage to the spinal cord and its elements;

mechanism of the trauma;

degree of wedge deformation of the vertebra;

post-traumatic instability of the vertebrae, which includes mechanical instability (type 1) characterized by abnormal spinal mobility, and neurological instability (type 2) caused by damage to the spinal cord and its elements induced by fragments of the fractured vertebra or by myelopathy developed in a distant period.

Considering the anatomy and the character of lesions of various spinal segments, we find it reasonable to analyze them separately. For example, to assess the severity of cervical spine injury, an examination is performed at the craniovertebral junction and the lower cervical spine [11].

Craniovertebral junction injuries:

$\mathrm{C}_{1}$ - the Jefferson bursting fracture;

$\mathrm{C}_{2}$ - the odontoid process fracture: apical - type 1 (conservative treatment), cervical - type 2, axis body fracture - type 3 (these types are subject to surgical treatment)

dislocations of $\mathrm{C}_{1}$ (the atlas) - anterior transligamentous; posterior and anterior odontoid;

dislocation-fracture of $\mathrm{C}_{2}$ (axis, hangman's fracture) a type of injury where the $\mathrm{C}_{2}$ arc breaks from both sides, the $\mathrm{C}_{2}-\mathrm{C}_{3}$ disk breaks up and $\mathrm{C}_{2}$ vertebra dislocates toward the front (surgical treatment is required).

A severity of lower cervical spine injury is scored using the Subaxial Cervical Spine Injury Classification (SLIC) [12].

A similar classification is developed for the Thoracolumbar Injury Classification System (TLICS) [13].

These systems are convenient because they allow one to assess the severity of the injury by scores, yet they are labor-consuming and not always suitable for emergency assistance.

Taking into account the existing ranking systems for SCVI, in 2013, the XXXVII Plenary Session of the Board of the Association of Neurosurgeons of Russia approved the Clinical Recommendations for the Treatment of Acute Complicated and Uncomplicated Spinal Injury in Adults.

The recommendations provide the assessment guidelines for spinal and spinal cord injuries, which consider:

the type of injury (isolated or combined);

the timing;

the nature (closed, open or penetrating injury);

the character (stable/unstable) and the mechanism of the injuries: compression (type A), distraction (type B), rotational (type $\mathrm{C}$ ), stabbing (type $\mathrm{K}$ ), gunshot or mine blast (type O);

the type of spine injuries: bruises, fractures, dislocations (including self-repaired), fractures, spondyloptosis, partial or full rupture of the capsularligament tissue in the vertebral-motor segment, intervertebrate disk rupture;

damage to the neurovascular structures of the spinal canal (uncomplicated or complicated injuries) and their category: concussion, contusion, compression, partial or full anatomical tear of the spinal cord and/or roots of the spinal nerves;

character of the compressing substrate: hematomas (sub- or epidural, intracerebral), bone fragments, traumatic disc hernia, foreign body; 
localization: cervical, thoracic, lumbar, sacral, multiple, multilevel, multiple multilevel lesions.

To optimize the diagnostic process and select the most suitable procedures for examination of SCVI patients, the understanding of advantages and disadvantages of the existing methods is of great importance.

\section{Materials and Methods}

We analyzed the causes and types of injuries as well as the diagnostic values of various imaging methods in 105 patients with acute and early stage spinal and spinal cord injury of different localizations: lumbar $(n=45$; $42.9 \%)$, thoracic $(n=17 ; 16.2 \%)$, cervical spine $(n=39$; $37.1 \%)$, and multi-vertebral injury $(n=4 ; 3.9 \%)$. The age of the patients varied from 21 to 70 years.

The study was conducted in accordance with the Helsinki Declaration (2013) and approved by the Ethics Committee of the Moscow State University of Medicine and Dentistry named after A.I. Evdokimov. An informed consent was obtained from each patient.

Upon admission, clinical and anamnestic data were evaluated, including the severity of neurologic damage (International Standards for Neurological Classification of Spinal Cord Injury, The American Spinal Injury Association (ASIA)); laboratory and imaging tests were then performed. Spine X-ray test was used in $100 \%$ of cases, and the novel technologies - in $75.2 \%$ of cases. Of them, MSCT (Toshiba Aquilion, Japan) under the standard protocol $-61.9 \%$ of cases, and MRI (Toshiba Vantage Atlas, Japan) with a magnetic field of 1.5 T. The standard MRI protocol included T2-weighted image (T2-WI) modes in three planes, T1-WI and STIR in the sagittal plane. If indicated the protocol was supplemented with $\mathrm{T} 1-\mathrm{WI}$ and $\mathrm{T} 2-\mathrm{Wl}$ images in the axial plane and T2 GE or T2* in the sagittal plane, as well as with magnetic resonance myelography.

In addition to the standard protocols (aimed to assess anatomic damage, spinal fluid abnormalities, and signs of edema in the bone marrow and spinal cord), additional methods were used to detect posttraumatic changes in the blood vessels (thromboses, aneurysms, dissections and intramural hematomas). For these purposes, the following ultrasound and tomographic techniques were used:

in patients with thoracic and lumbosacral injuries CT angiography;

in patients with cervical spine damage - magnetic resonance angiography (MRA); to obtain information about anatomy of the arteries - the 3D time-of-flight technique (TOF) was used; if the resulting angiograms were of poor quality or if additional information was needed, a novel technology of time-spatial labeling inversion pulse (Time-SLIP) was applied;

to visualize the efferent vessels and draining venous reservoirs, we used the two-dimensional time-of-flight technique (2D TOF).

In 32 patients, the imaging findings were verified during surgery. In 73 patients with stable vertebral fractures without significant deformity and neurological damage (when surgical interventions were contraindicated), the verification analysis was limited to dynamic observation. Control spine X-ray, MRI, and MSCT tests were conducted at various time points to assess the treatment efficacy.

In the course of the study, the following diagnostic tasks were addressed:

to assess the level and extent of spine injury, the type and nature of the fractures, their stability and instability;

to reveal the state of intervertebral discs;

to identify the localization and type of damage to the spinal cord, ligaments, and vessels or the potential damage to these structures in the case of instability.

\section{Results and Discussion}

Among the causes of spine and spinal cord injury, a strong external impact occurred most often. In a third of cases $(n=34 ; 32.4 \%)$ the impact was caused by traffic accidents involving vehicles: cars, buses, motorcycles etc., driving at a speed of $60 \mathrm{~km} / \mathrm{h}$ and more; in $24.3 \%$ of cases $(n=26)$ the injury followed a fall from a height of $>1 \mathrm{~m}$ and a significant axial load.

Of the injury mechanisms, the most common were compression $(n=51 ; 48.5 \%)$ and flexural $(n=25 ; 23.8 \%)$ injuries; the rarest were extensor lesions $(n=6)$, extensor lesions with disco-ligament tears caused by dislocations $(n=5)$ or excessive stretching $(n=3)$.

There were a few puzzling situations where the risk of SCVI increased and making the right diagnosis became difficult:

degenerative changes and osteoporosis (in all patients older than 65 years, $n=10 ; 9.5 \%$ );

other collateral disorders, including primary and secondary neoplastic processes, metabolic disorders, coagulopathies $(n=21 ; 20.0 \%)$ and spinal abnormalities $(\mathrm{n}=8 ; 7.6 \%)$ (Figure 1);

"distracting" factors ( $n=9 ; 8.6 \%)$, e.g. a reduced level of consciousness in patients with head injury or under the influence of alcohol or drugs.

The present analysis of diagnostic significance, possibilities, and limitations of the imaging methods showed that in $23.8 \%$ of cases $(n=25)$, vertebral fractures were not detected in spine X-ray images but only with MSCT or MRI scans. Largely, this observation pertained to damages to the cervical and thoracic areas, where the summation effects were most pronounced and the incidence of unidentified fractures reached $54.3 \%$ $(n=29)$. Spine $X$-ray failed to identify vertebral contusion, stress fractures, minor lesions, small bone fragments, and the severity of compression or damage to the spinal cord and the neural structures.

In $97.1 \%$ of cases, MSCT was able to detect bone trauma (that allowed us to describe and characterize it), damage to the brain, the thoracic and abdominal internal organs. 

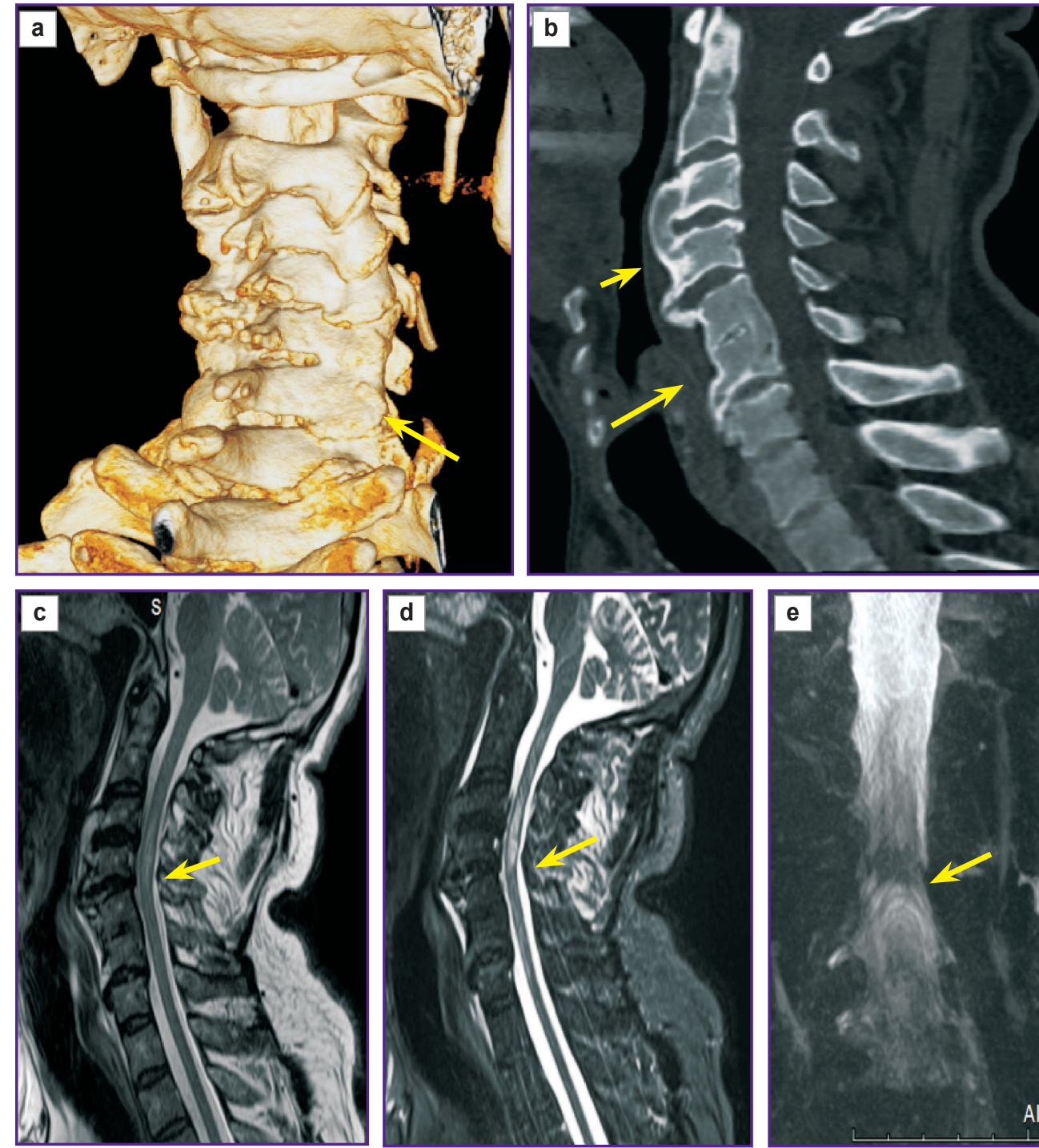

Figure 1. MSCT: (a) 3D reconstruction, (b) sagittal projection; MRI: (c) T2-WI, (d) STIR in sagittal projections; and myelogram (e) of patient B., 48 years old, who presented with tetraparesis after tilting the head during exercise

MSCT showed concrescence of vertebrae $\mathrm{C}_{5}$ and $\mathrm{C}_{6}$, abnormal kyphosis, stenosis of the spinal canal, osteochondrosis, and spondylosis of the cervical spine - arrows in (a) and (b). Due to the discrepancy between the clinical and radiological data, an MRI scan was performed, which revealed signs of spinal cord injury and spinal cord concussion with a hemorrhagic component at the $\mathrm{C}_{3}-\mathrm{C}_{5}$ level - arrows in (c) and (d). In the magnetic resonance myelograms - notable absence of cerebrospinal fluid flow at the level of spinal stenosis $\left(\mathrm{C}_{4}\right.$ and $\left.\mathrm{C}_{5}\right)-\operatorname{arrow}$ in $(\mathrm{e})$

The disadvantages of MSCT include high radiation power, insufficient diagnostic capability of describing traumatic myelopathy and determining a compressive substrate in the spinal cord injury: among them, disc herniation, ligament fragments, epidural, sub- and intracerebral hematomas (Figure 2).

In all cases, during the MSCT images processing, the $2 \mathrm{D}$ and $3 \mathrm{D}$ reconstructions were performed; those brought an important diagnostic value to the detection of compression and rotational trauma components, which could be hardly diagnosed in the axial slices. Thus, in patients with multiple multilevel and combined injuries, MSCT can be considered as the method of choice (without running of standard X-ray tests). The use of the "Polytrauma" (Whole body) protocol provided the most impressive results. Therefore, we find it optimal to use the following scanning procedures:

with no intravenous contrast enhancement - the skull and brain, the cervical spine - from the craniovertebral junction to the upper thoracic region $\left(\mathrm{Th}_{4}\right)$;

with intravenous contrast enhancement (multiphase or the "split bolus" technique) - the thoracic and 
Figure 2. MSCT (a) and MRI (b) images made in the axial projection; sagittal MRI images: (c) T1-WI, (d) T2-Wl; and myelogram (e) of patient S., 28 years old, pregnancy 38 weeks, presented with tetraparesis after a minimal external impact on the cervical and upper thoracic spine on the background of direct anticoagulant therapy

None of the tomographic scans found any vertebral lesions. An extradural hemorrhage with dorsolateral localization was detected at the $\mathrm{Th}_{1^{-}}$$\mathrm{Th}_{3}$ level (arrows), also notable is a compressed dural sac and a spinal cord with no cerebrospinal fluid flow at $\mathrm{Th}_{2}$ and $\mathrm{Th}_{3}$ levels
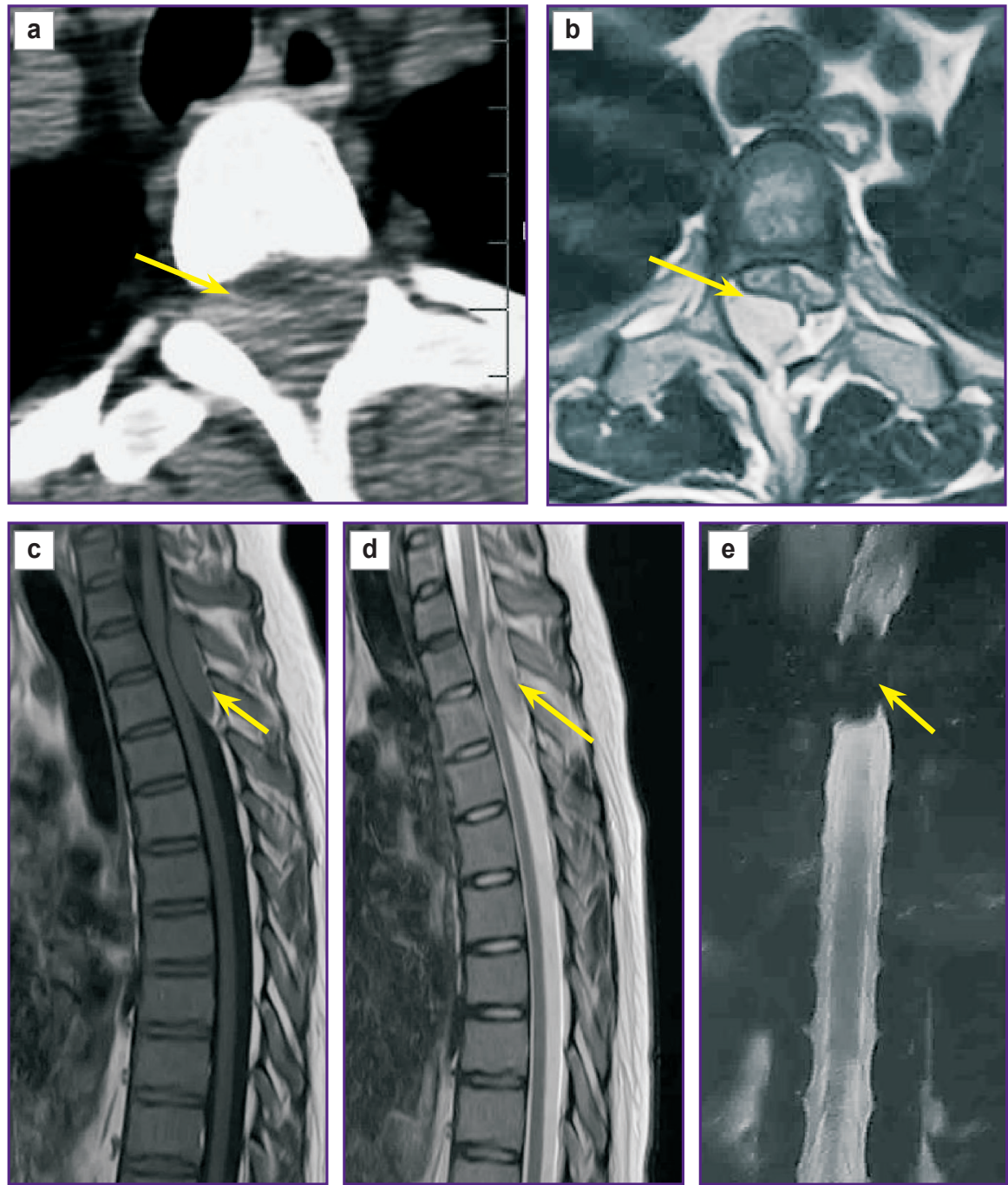

abdominal cavities; this approach allows one to use different filters in order to analyze blood vessels, internal organs, ribs, pelvis, spine, the contours of the vertebrae, their structures and interconnections, as well as the configuration of the spinal canal (Figures 3, 4).

To detect changes in the vertebral arteries, the method of CT angiography is not very effective because of the small diameter of these blood vessels and significant artifacts caused by the surrounding bone structures. In the present study, MRA proved to be a more effective modality for the diagnosis of cervical blood vessel lesions. The MRA was performed in $17.5 \%$ of cases and allowed us to reveal dissections of the vertebral artery in V2 and V3 segments in 5\% of patients with cervical vertebral fractures. The frequent damages to the vascular wall in these segments can be explained by considerable mobility of the arteries at these levels and their anatomical proximity to bone structures.

MRA allows one to evaluate abnormalities in the blood flow, e.g. the presence of no-signal zones, changes from laminar to turbulent flow, as well as signs of dissection like the extended uniform "string" symptom, an uneven stenosis, the "wavy ribbon" tortuosity of the blood vessel, and the signs of intramural hematomas (Figure 5). In this study, MRA was performed in addition to the standard protocol; the latter was sensitive enough to detect changes in the bone marrow and describe the localization, character, and extension of spinal and soft tissue injuries (spinal cord, spinal nerves, intervertebral discs, ligaments) in $95.2 \%$ of cases.

The use of MRI in emergency situations is limited by the guidelines, which do not recommend using this method for primary diagnostic procedures performed in urgent cases; therefore, MRI is not included in the medical standards of emergency care in patients with multiple trauma.

MRA is a lengthy examination that requires the patient to remain steady during the scan, which limits MRA implementation in emergency situations. In the cases of neurological deficit in patients with SCVI, in stable patients, and in patients with no contraindications, MRI of the spine and spinal cord may be performed within the first $6-8 \mathrm{~h}$ after the trauma in $16.2 \%$ of cases, and 

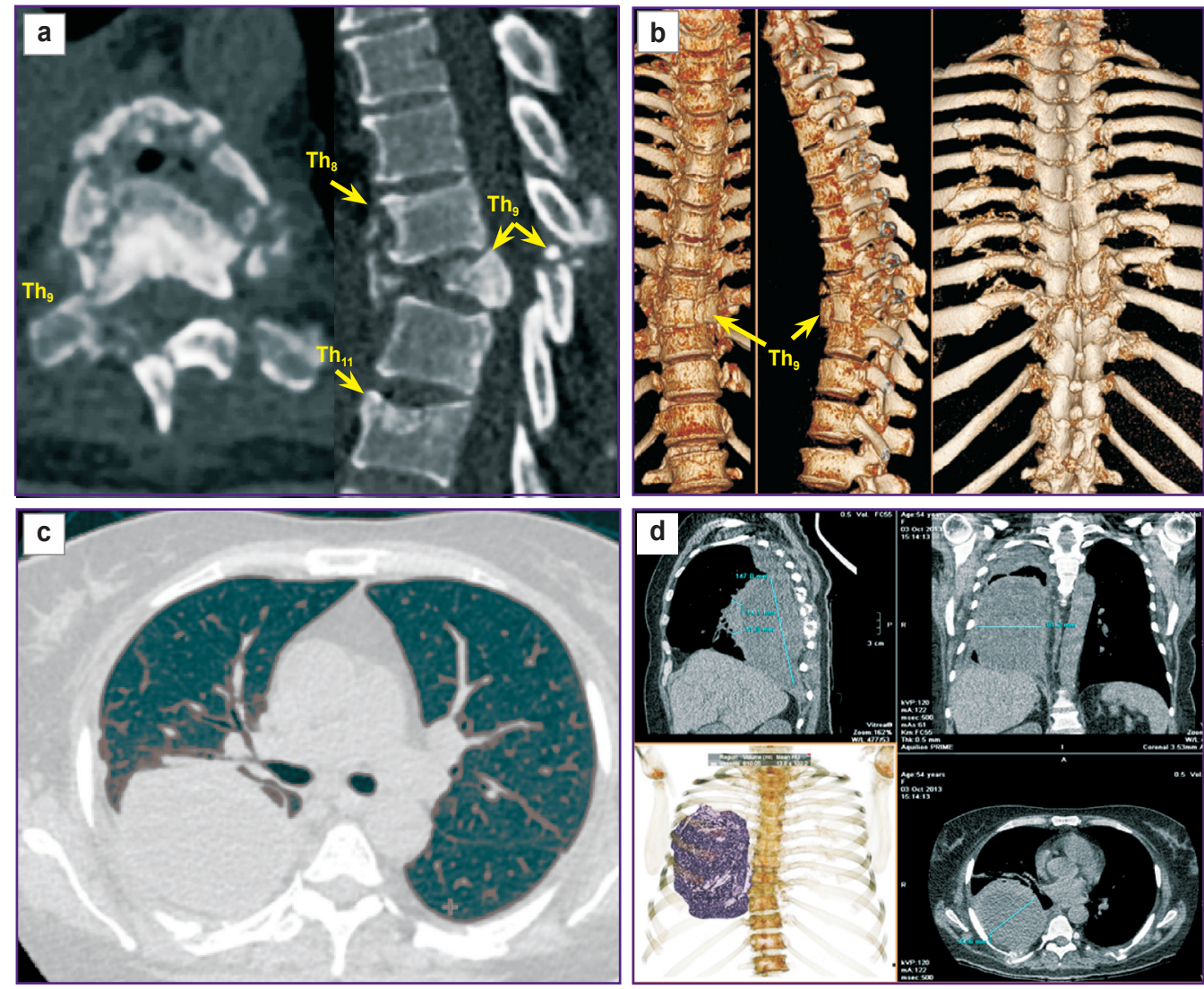

Figure 3. MSCT of the chest and thoracic spine of patient G., 23 years old, with polytrauma caused by a traffic accident:

(a) thoracic vertebrae in the bone window, in the axial and sagittal projections; (b) 3D reconstruction of the thoracic spine and ribs; (c) thoracic cavity in the lung window, axial projection; (e) multiplanar and 3D-reconstructed images of the thoracic cavity in the soft tissue window. There are multiple bilateral rib fractures along the scapular and paravertebral lines with a displacement of fragments, an "explosion" $T h_{9}$ fracture with an extension of the fracture line from the vertebral body to the arc and articular processes, with the displacement of fragments of the vertebral body toward under the anterior longitudinal ligament and the spinal canal. Also notable are: spinal stenosis, abnormal kyphosis, and impression fractures of $\mathrm{Th}_{8}$ and $\mathrm{Th}_{11}$ vertebral bodies - arrows in (a), (b). The fractures are complicated by a concussion and rupture of the right lung with right-side hydrothorax (according to the density characteristics, it is blood)
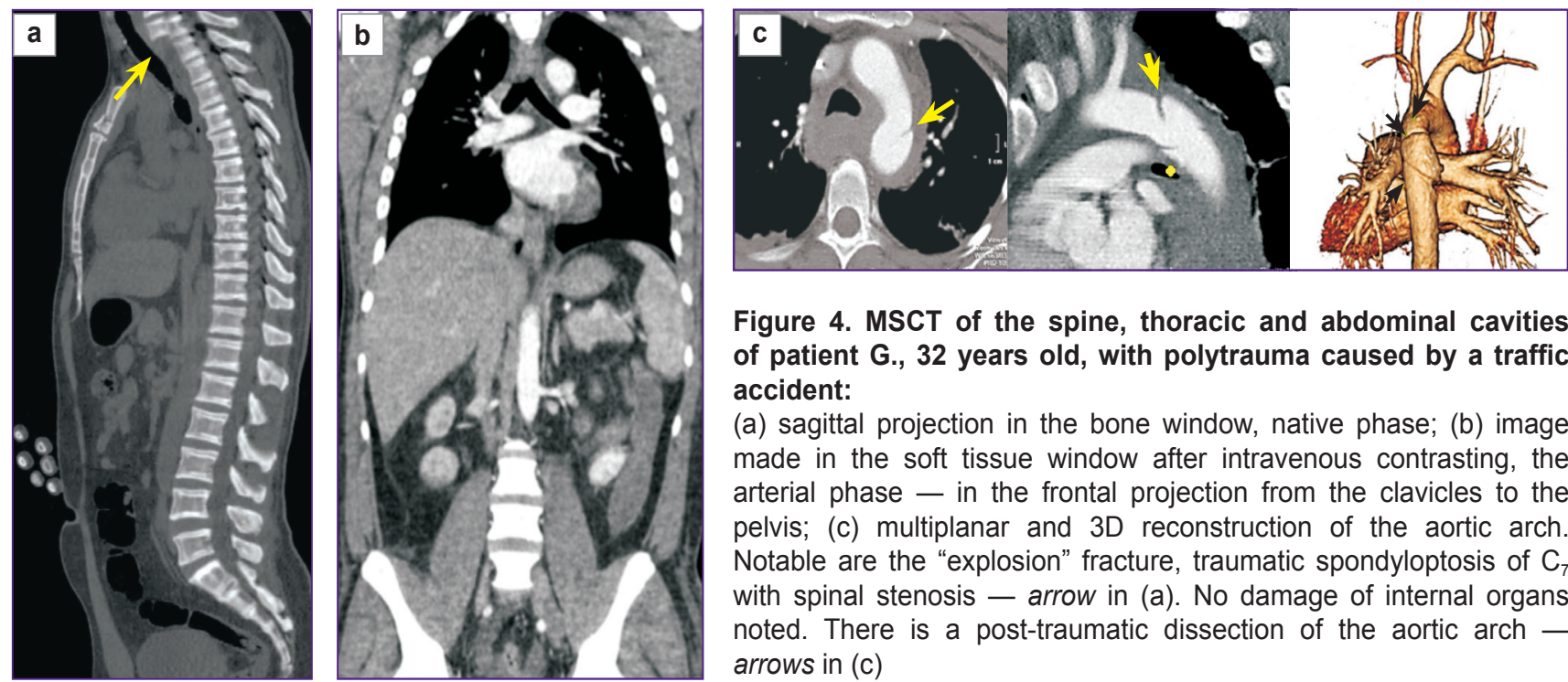

Figure 4. MSCT of the spine, thoracic and abdominal cavities of patient G., 32 years old, with polytrauma caused by a traffic accident:

(a) sagittal projection in the bone window, native phase; (b) image made in the soft tissue window after intravenous contrasting, the arterial phase - in the frontal projection from the clavicles to the pelvis; (c) multiplanar and 3D reconstruction of the aortic arch. Notable are the "explosion" fracture, traumatic spondyloptosis of $\mathrm{C}_{7}$ with spinal stenosis - arrow in (a). No damage of internal organs noted. There is a post-traumatic dissection of the aortic arch arrows in (c) 

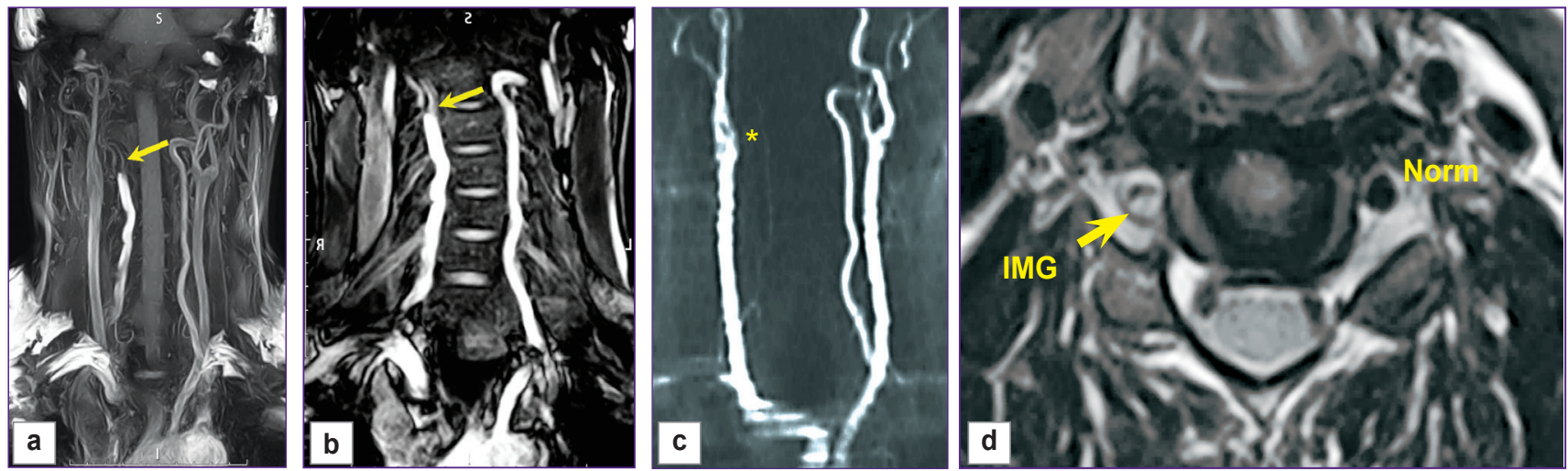

Figure 5. Cervical spine MRI in patient $\mathrm{K}$., 28 years old, with polytrauma resulted from a traffic accident

In the frontal plane: (a) Time-SLIP; (b) 3D TOF; (c) 2D TOF. In the axial plane: (d) T2-WI, signs of decreased flow and dissection of the right vertebral artery wall in segment $\mathrm{V} 2$ (at the level of $\mathrm{C}_{3}-\mathrm{C}_{5}$ bodies). In the 3D TOF and Time-SLIP images, the artery looks excessively tortuous with the positive "string" symptom (thin arrows). The artery is not visualized in the 2D TOF mode (asterisk); in segment $\mathrm{V} 3$ on T2-WI, there is no flow artifact due to a decrease in blood flow and presence of IMG (thick arrow)

within 3 days - in $74.3 \%$. These time points are most significant in terms of identifying:

X-ray negative fractures of the vertebrae (stress and latent);

injuries of intervertebral discs and ligaments (ruptures, traumatic hernias);

neurological damage, injury of the spinal cord, neural structures and membranes;

the level, degree, and cause of stenosis of the intervertebral foramen and the spinal canal.

Timely detection of these changes is necessary to determine the indications for surgical intervention, which may be needed to examine the spinal cord and nerve roots, their decompression, stabilization in cases of unstable fractures, and subarachnoid cerebrospinal fluid space that may be aggravated by instability. In determining the severity of stenosis, the sagittal size and the area of the spinal canal are taken into account. The most informative parameter is the relation between the compressing agent and the neural structures (see the Table).

It should be noted that in the acute period it is difficult, and in $4.8 \%$ of cases, it is impossible, to discern between a spinal cord tear, contusion, and an intramedullary hematoma. These lesions can be diagnosed more precisely after regression of edema/hemorrhage or under the condition of spinal shock. If the continuity of the spinal cord preserves and the above changes persist for up to 30 days, they are usually associated with neurological deficit, atopic paralysis, areflexia, anesthesia, dysfunction of the pelvic organs, and trophic disorders.

Based on the results of the study, and considering the possibilities and limitations of the imaging methods, we proposed an examination procedure for patients with SCVI (Figure 6).

The use of the proposed scheme helps one to take timely clinical decisions, select the treatment, and predict the injury outcome.
Compression of neural structures

(graded according to Pfirrmann et al. [14]

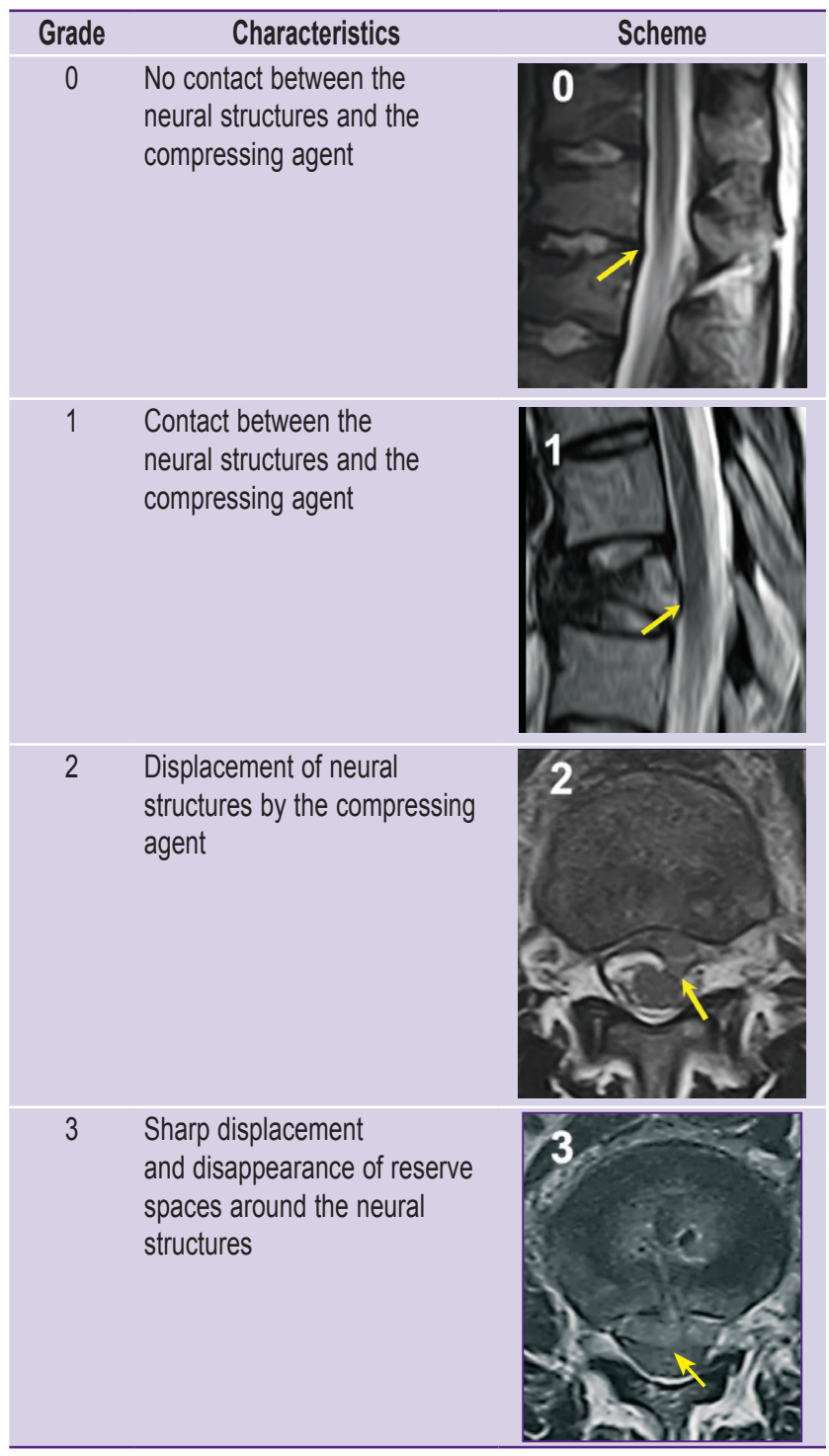




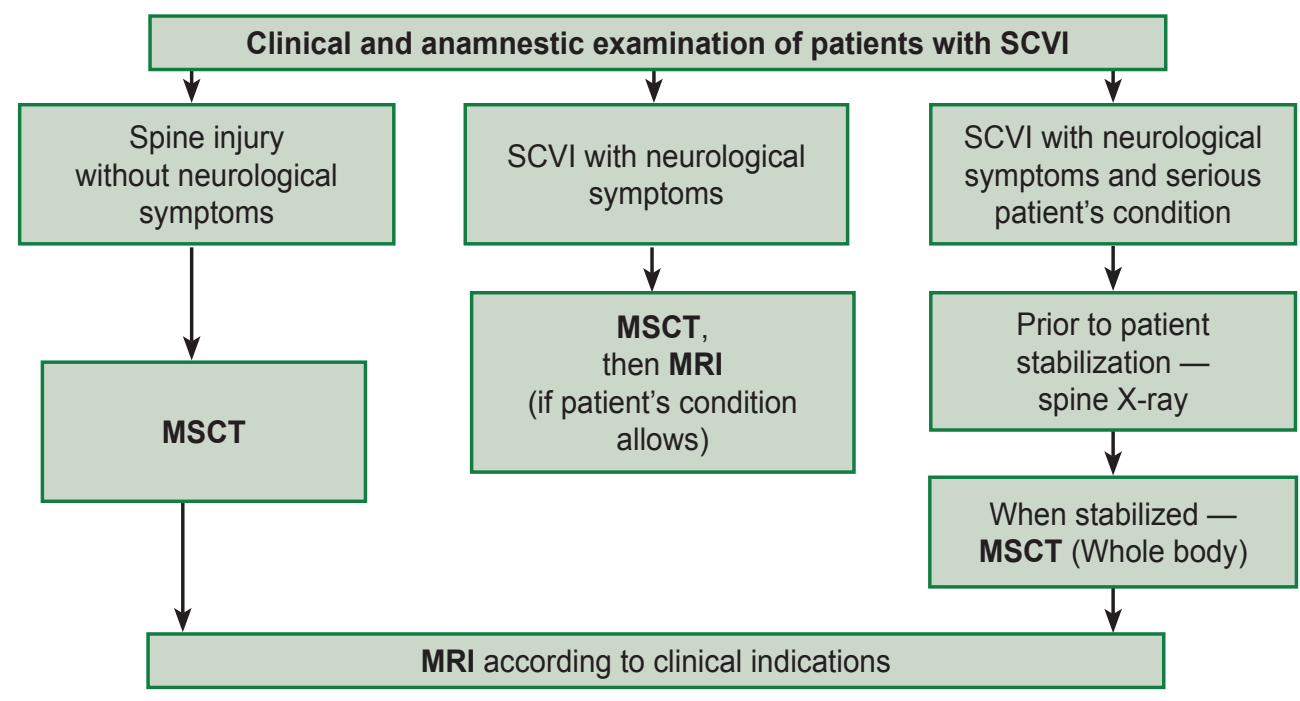

Figure 6. Schematic representation of the imaging diagnostic procedures in patients with spinal cord and vertebrae injury (SCVI)

MSCT represents the method of choice in the diagnostic procedure for combined injuries of the spine, skull, brain, chest and abdominal cavity. In the presence of neurological symptoms and suspected damage to the spinal cord or the neural structures, MRI (with magnetic resonance myelography and MRA) should be included in the examination protocol.

However, in the case of multiple multilevel and combined injuries under severe patient's conditions, the imaging can be limited to spine X-ray. After stabilization of patient's condition, MSCT should be performed according to the Whole body protocol with intravenous contrast enhancement. MRI should be performed in a more distant period according to clinical indications. Other authors from the Russian Federation proposed the similar diagnostic schemes for SCVI patients $[15,16]$.

\section{Conclusion}

In acute spinal trauma under the conditions of emergency, the most rational approach is to conduct a comprehensive imaging examination using the novel diagnostic technologies.

In suspected spinal cord and vertebrae injury, it is risky to limit the examination by a single diagnostic method because some important information on the spine and spinal cord can be lost.

In all patients with complicated trauma and neurological symptoms, the preferable method at the primary stage is MSCT followed by mandatory MRI at the earliest possible time.

Financial support. The study was not funded by any sources.

Conflict of interests. The authors declare that the study, its subject and content do not involve conflicting interests.

\section{References}

1. Krylov V.V., Grin' A.A., Lutsik A.A., Parfenov V.E., Dulaev A.K., Manukovskii V.A., Konovalov N.A., Perl'mutter O.A., Safin Sh.M., Kravtsov M.N., Manashchuk V.I., Rerikh V.V. A protocol recommended for treating acute complicated and uncomplicated spinal injuries in adult patients (Association of Neurosurgeons of Russia). Part 1. Voprosy neirokhirurgii imeni N.N. Burdenko 2014; 78(6): 60-68, https:// doi.org/10.17116/neiro201478660-67.

2. Marinchek B., Dondelinger R.F. Neotlozhnaya radiologiya. Chast' 1. [Emergency radiology. Vol. 1]. Moscow; VIDAR-M; 2008; 342 p.

3. Cassar-Pullicino V.N., Imhof H. Spinal trauma - an imaging approach. Thieme; 2008; 256 p.

4. Grin' A.A., Grigoreva E.V. The radiology diagnostics of vertebral and spinal trauma. Part 1. Neirokhirurgiya 2012; 4: 8-16.

5. Grin' A.A., Grigoreva E.V. The radiology diagnostics of vertebral and spinal trauma. Part 2. Neirokhirurgiya 2013; 1 : $7-21$.

6. Aganesov A.G. The future and the past of surgery for the complicated spine trauma. Khirurgiya. Zhurnal imeni N.I. Pirogova 2013; 1 : 5-12.

7. Schedrenok V.V., Yakovenko I.V., Moguchaya O.V., Orlov S.V., Anikeev N.V. Associative craniocerebral and vertebral-cerebrospinal trauma in cities with different number of population. Nevrologicheskiy vestnik. Zhurnal imeni V.M. Bekhtereva 2007; 2: 77-79.

8. Denis $F$. The three column spine and its significance in the classification of acute thoracolumbar spinal injuries. Spine 1983; 8(8): 817-831, https://doi.org/10.1097/00007632198311000-00003.

9. Saldun G.P. Zadniy kombinirovannyy spondilodez $i$ ego mesto $v$ operativnom lechenii tyazhelykh perelomov grudnogo i poyasnichnogo otdelov pozvonochnika. Dis. ... 
kand. med. nauk [Rear combined spondylodesis and its place in the surgical treatment of severe fractures of the thoracic and lumbar spine. PhD Dissertation]. Leningrad; 1983.

10. Magerl F., Aebi M., Gertzbein S.D., Harms J., Nazarian S. A comprehensive classification of thoracic and lumbar injuries. Eur Spine J 1994; 3(4): 184-201, https://doi. org/10.1007/bf02221591.

11. Anderson L.D., D'Alonzo R.T. Fractures of the odontoid process of the axis. J Bone Joint Surg Am 1974; 56(8): 16631674; https://doi.org/10.2106/00004623-197456080-00017.

12. Patel A.A., Dailey A., Brodke D.S., Daubs M., Anderson P.A., Hurlbert R.J., Vaccaro A.R.; Spine Trauma Study Group. Subaxial cervical spine trauma classification: the Subaxial Injury Classification system and case examples. Neurosurg Focus 2008; 25(5): E8, https://doi.org/10.3171/ foc.2008.25.11.e8.

13. Andrei F.J., Michael D.D., Brandon D.L., Brodke D.S.,
Cendes F., Tedeschi H., Patel A.A. Retrospective evaluation of the validity of the Thoracolumbar Injury Classification System in 458 consecutively treated patients. Spine 2013; 13(12): 1760-1765, https://doi.org/10.1016/j.spinee.2013.03.014.

14. Pfirmann C.W., Dora C., Schmid M.R., Zanetti M., Hodler J., Boos N. MR image-based grading of lumbar nerve root compromise due to disk herniation: reliability study with surgical correlation. Radiology 2004; 230(2): 583-588, https:// doi.org/10.1148/radiol.2302021289.

15. Sedelnikov S.S., Dorovskikh G.N. Whole-body multidetector computed tomography as a stage of early diagnosis of polytrauma (review of literature and own experience). Radiologiya - praktika 2017; 5(65): 22-30.

16. Dorovskikh G.N., Gorlina A.Yu. Radiologic evaluation and polytrauma treatment according to reports ATLS (review of the literature and own observations). Radiologiya - praktika 2017; 5: 73-81. 